\title{
Functionalized coatings by Cold Spray: an In Vitro Study of micro- and nanocrystalline
} Hydroxyapatite compared to Porous Titanium

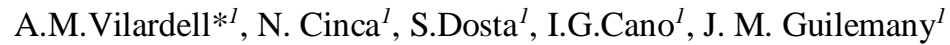 \\ X.Nogués ${ }^{2}$, N.Garcia-Giralt ${ }^{2}$ \\ ${ }^{1}$ Centre de Projecció Tèrmica (CPT). Dpt. Ciència dels Materials i Enginyeria Metal.lúrgica. Universitat \\ de Barcelona Martí i Franquès 1, 08028 Barcelona, Spain. \\ ${ }^{2}$ IMIM (Institut Hospital del Mar d'Investigacions Mèdiques), RETICEF, CIBERFES, ISCIII. Doctor
}

Aiguader 80, 08003 Barcelona, Spain

*Corresponding author: amvilardell.research@gmail.com

\begin{abstract}
Three different surface treatments on a Ti6Al4V alloy have been in vitro tested for possible application in cementless joint prosthesis; all of them involve the novelty of using the Cold Spray technology for their deposition: (i) an as-sprayed highly rough titanium and, followed by the deposition of a thin hydroxyapatite layer with (ii) microcrystalline or (iii) nanocrystalline structure. Primary human osteoblasts extracted from knee were seeded onto surfaces and cell viability using MTS and LIFE/DEAD assays, osteoblasts differentiation by alkaline phosphatase (ALP) quantification as well as cell morphology were tested at 1, 7 and 14 days of cell culture.

Different cell morphologies between titanium and hydroxyapatite surfaces were exhibited; at 1 day of cell culture, cells on the titanium coating were spread and flattened, expanding the filopodia actin filaments in all directions, while cells on the hydroxyapatite coatings showed round like-shape morphology due to a slower attachment. Higher cell viability was detected at all times of cell culture on titanium coating due to a better attachment at 1 day. However, from 7 days of cell culture, cells on hydroxyapatite showed good attachment onto surfaces and highly increased their proliferation, mostly on nanocrystalline, achieving similar cell viability levels than titanium coatings. ALP levels were significantly higher in titanium, in part, because of greatest cell number. Overall, the best cell functional results were obtained on titanium coatings whereas microcrystalline hydroxyapatite presented the worst cellular parameters. However, results indicate that nanocrystalline hydroxyapatite coatings may achieve promising results for the faster cell proliferation once cells are attached on the surface.
\end{abstract}

Keywords: Titanium; Hydroxyapatite; Cold Spray; in-vitro testing;

\section{INTRODUCTION}

The surface treatments for cementless joint prosthesis have been the subject of exhaustive study during the last decades. These have been performed with the intention to improve the osseointegration of titanium aiming at modify the microstructure of the surface by texturing or changing the chemical composition [1]; textures have played an important role leading to an increase of free surface and cell differentiation [2]. On the micron scale, roughness is clearly important and it has been shown to be important for bone bonding. However, those textures should be in a range between 100-500 microns to enable the bone ingrowth for good mechanical fixation [3]. Moreover, textures are also important at micro/nanoscale; surface treatments 
such as grit-blasting and acid-etching [4,5] and anodization [6,7], enhance cell response. However, surface patterns have yet to be established. By these approaches, the ability of the prostheses to bind to bone is obtained through a new morphology, an improved roughness and a bigger contact area between the implant ant the tissue. Surface treatments can also involve chemical modifications on Ti such as the deposition of bioactive ceramics as could be calcium phosphates or bioactive glasses [8,9]. Those treatments show significant effects in-vitro and in-vivo [10]. There exists an extensive work in the literature dealing with the application of calcium phosphates as bioactive layers by many several methodologies, especially concerning hydroxyapatite (HA) due to their osteogenic properties and the ability to form strong bonds with host bone tissues [11]. The bioactivity and biodegradation of HA are critical parameters to achieve good HA coatings that mainly depend on HA phase and crystallinity [12].

The most commonly well-established process for commercial prosthesis is Plasma Spraying (PS), either covering the surface implants with titanium porous surfaces or with stoichiometric hydroxyapatite. In the case of titanium coatings, the Vacuum Plasma Spraying (VPS) is mostly used to avoid oxidation. With regard to HA coatings, they are reported to lead to an early new bone formation on their surface but lately few concerns have appeared discussing their improvement effect [12]. Plasma sprayed HA coatings have shown long term failures related to the weak adhesion of HA coating due to the formation of Amorphous Calcium Phosphate (ACP) phases caused by the high flame temperatures [12]. Hiler et al. [13] reported that the use of HA coating on stems available both with and without this surface treatment had no clinically relevant effect on their outcome. Contrarily, early follow-up showed that HA cementless acetabular cup implant design exhibits high survivorship and clinical success with lower incidence of loosening and migration than older designs [14]. Findings suggested inferior survival HA-coated by premature failure due to the disintegration of the particles of the coating, resulting in an increase of osteolysis and aseptic loosening failures [15]. Lower temperature techniques such as the High Velocity Oxygen Fuel (HVOF) process were also studied, and although higher crystalline coatings were obtained in comparison with PS, it was still observed the formation of ACP phase at the interface coating/substrate [12,16, 17], detrimental for a suitable bond strength of the coating in vitro. $100 \%$ crystalline coatings were obtained after a thermal treatment at $700{ }^{\circ} \mathrm{C}$ for $60 \mathrm{~min}$, leading to a bond strength after immersion in Simulated Body Fluids (SBF) much higher than without treatment [17]. Different HA coatings from less to high crystallinity were invitro evaluated with human osteoblast cells [18]. Results showed that different phases do not affect cell viability and cell proliferation but the presence of ACP induces a higher cell differentiation.

Cell response depends on surface parameters such as topography, chemistry and structure [19]. Topographical surface changes as could be roughness and porosity induce good cell response [20]. Results suggest that the introduction of nanoscale structures in combination with micro-/submicro-scale roughness improves osteoblast differentiation and local factor production, which, in turn, indicates the potential for improved implant osseointegration in vivo [21]. Surface chemistry, as could be formation of sodium titanate hydrogel on the surface of the implant due to an alkaline treatment [22] or the deposition of calcium phosphates such HA [9], should allow for earlier load bearing of prostheses following implantation. Specifically, HA surfaces seem desirable due the induction of bone like apatite formation, which is considered to be a reason for better osseointegration [23]. Concerning the structure, cells are sensitive to small surface features; Fernandes et al. [24] demonstrated that the use of small grain sizes, as could be ultra-fine grained Ti particles, induces favorable biological responses, as well as the used of nanocrystalline 
HA, a topic of special interest due its similar crystal bone size microstructure. Nanocrystalline HA provided high surface area which induced better bioactivity than coarse crystals [25]. Webster et al. [26] reported that osteoblast functions on ceramics with grains smaller than $100 \mathrm{~nm}$ were significantly enhanced compared with larger grains. So, the production of nanocrystalline HA coatings on titanium seem desirable for implants if it can be accomplished at low temperature.

The Cold Spray (CS) coating technology has been suggested as a good alternative to spray oxygen- and temperature-sensitive materials, due to its low temperature range in comparison with the conventional thermal spray technologies [27]. CS is a solid-state process based on the kinetics energy and stands above conventional spray techniques for its low temperature rates. Small particles $(5-50 \mu \mathrm{m})$ are accelerated by a pre-heated gas temperature $\left(25-1100^{\circ} \mathrm{C}\right)$ lower than the melting point of the material and propelled toward a substrate at supersonic velocities $(300-1200 \mathrm{~m} / \mathrm{s})$. Since particles are not melted for cold spray, the resulting deposits exhibit moderate compressive residual stress. CS started by the spraying of ideal metallic materials with relatively low melting points and lower mechanical strengths such as $\mathrm{Zn}, \mathrm{Al}$ and $\mathrm{Cu}$, to higher strength materials such as Ti and the corresponding alloys, as well as tantalum and even cermet and ceramic materials $[28,29,30]$. Recently, several coating systems have been obtained through this process for biomedical purposes [31]. Both, titanium and calcium phosphate coatings have been lately produced [9, $27,32,33,34]$. However, very few works deal with their performance in vitro and in vivo [10,35]. CS calcium phosphate attempts were mostly produced on soft substrates such as PEEK [10], in which facilitates the differentiation and the proliferation of cultured cells, or magnesium [36] in which was improved the biodegradability of $\mathrm{Mg}$ alloy attributed to the dissolution and re-precipitation of apatite. The soft substrates embedded ceramic HA particles embedded into them leading to HA coatings. However, recently attempts of calcium phosphate coatings were obtained onto hard substrates such as Ti6Al4V alloy, in which powder structure plays an important role on its deposition [9,32,34].

A comparative study between Ti and HA coatings obtained by APS and VPS has been carried out [37]. In both cases, VPS is recommended as a better method to deposit Ti and HA coatings that can be applied as hard tissue replacement implants. This work was especially interesting in the sense that provides a comparison in the biological performance between such coatings having different composition and surface morphology obtained by the conventional technologies. Usually, it is quite difficult to justify the cell behavior when such different variables are considered. A similar work is here presented but for cold sprayed coatings. Currently, very few biological studies of CS HA coatings have been performed, due to the challenge to spray ceramic particles by CS [10].

In view of what has been exposed, the aim of the present article is to present an in vitro comparison of (i) an as-sprayed highly-rough titanium coating with two others: one followed by the deposition of a thin hydroxyapatite layer with microcrystalline feedstock powder and the other with nanocrystalline feedstock powder; all the coatings are deposited by CS. The results will be presented and discussed according to the specific spraying conditions here employed and cannot be generalized. 


\section{MATERIALS AND EXPERIMENTAL METHOD}

\subsection{Sample preparation and surface characterization}

A Commercial Pure grade 2 Titanium (CP-Ti) powder with irregular shape was used for that purpose. CPTi particles present the typical morphology obtained by attrition process, with a particle size between 90 $150 \mu \mathrm{m}$. The particles exhibit an irregular morphology with angular shapes (Fig. 1a), showing a dense and a compact microstructure; also, two different HA powders with different microstructures were chosen: (i) a microcrystalline HA (C-HA) powder obtained by sintering from Plasma-Biotal Ltd. (Captal30, Derbyshire, UK) and (ii) an agglomerated nanocrystalline HA (NC-HA) powder from Medicoat (France). Both HA powders present spherical morphologies. NC-HA powder has a particle size distribution between $63-40 \mu \mathrm{m}$ while C-HA of 40-20 $\mu \mathrm{m}$. Figure $1 \mathrm{~b}$ shows the spherical morphology of the agglomerate NC-HA particle composed of very fine agglomerates whereas figure 1c displays the morphology of sintered structure showing a porous morphology formed by microcrystalline grains. The morphological characterization of the feedstock powders was performed by Scanning Electron Microscopy SEM (ProX Phenom) equipped with an EDS for microanalysis. All powders were sprayed onto Ti6Al4 alloy substrates of $2 \mathrm{~mm}$ thickness and $7 \mathrm{~mm} \times 7 \mathrm{~mm}$ dimension size for in-vitro tests.

The CP-Ti coatings were previously optimized with CGS KINETICS ${ }^{\circledR} 4000$ with a maximum operating gas pressure of 40 bars, gas temperature of $800^{\circ} \mathrm{C}$ and with nitrogen as the propellant gas. C-HA and NCHA powders were afterwards deposited onto CP-Ti coatings by CS [9,33,34].

Surface examinations were performed by Field Emission Scanning Electron Microscopy (FESEM JEOL JSM 7100F). The cross-section areas were prepared by grinding and polishing samples until $1 \mu \mathrm{m}$ size diamond suspension, and the coating thickness values were measured according to ASTM F1854 with optical microscopy (Leica DMI5000 M). The phase identification of HA coatings was analyzed by X'Pert PRO MPD diffractometer (PANalytical) with $\mathrm{Cu} K \alpha$ radiation. The roughness of the coatings was measured by confocal microscopy (Leica DCM3D) and the results were presented with the Sa-Sz and Ra-Rz global values for the 3D surface and 2D profile as well as the $\mathrm{Ra}$ and $\mathrm{Rz}$ of the 2D profile separated in the waviness and microgroughness contribution according to ISO 4287, at a magnification of 10x and an stitching of $2 \times 2$. The surface nanoroughness of the coatings was measured by Multimode8 AFM with Nanoscope V (Bruker) controller and a SNL (Bruker) probe $(\mathrm{K}: 0.35 \mathrm{~N} / \mathrm{m})$. A custom-made water contact angle goniometer with imageJ software program was used to the measurement of the contact angles, performing static measurements in sessile drop mode with $2 \mu 1$ droplet of MilliQ water.

\subsection{Cell culture}

Human osteoblastic cells (HOBs) have been obtained from knee trabecular bone of postmenopausal women undergoing knee replacement due to osteoarthritis, following the protocol described by Nacher et al. [38]. The entire study has been approved by the Parc de Salut Mar Ethics Committee. Briefly, trabecular bone was dissected into small pieces, thoroughly washed in phosphate-buffered solution (PBS) and placed into a $15 \mathrm{~cm}$ diameter Petri dish containing $15 \mathrm{ml}$ Dulbecco's modified Eagle's medium (DMEM) supplemented with $10 \%$ FBS, penicillin (100 UI/ml), streptomycin (100 UI/mL), ascorbic acid (100 $\mu \mathrm{g} / \mathrm{ml})$ (Invitrogen) and fungisone $(0.4 \%)$ (Gibco). The explants were incubated at $37{ }^{\circ} \mathrm{C}$ in a humidified atmosphere of $5 \%$ $\mathrm{CO}_{2}$, changing the medium once a week until cell confluence. Finally, cells were subcultured into new 75 
$\mathrm{cm}^{2}$ flasks until the needed cell number has been reached. A maximum of a third subculture has been used in the experiments. For materials testing, samples were overnight sterilized in ethanol $70^{\circ}$, washed in PBS and placed on a 48-well polystyrene culture plate (Nunc A/S). Each material was seeded with 100.000 cells and cultured with DMEM supplemented with $10 \%$ FBS and ascorbic acid. Samples were tested at 1, 7 and 14 days of cell culture. Tests were carried out three times in order to ensure reproducibility. Each test contained two replicas of each sample and was tested together positive and negative controls. Results were normalized by the titanium samples within each experiment and each time in order to avoid the interexperiment variability.

\subsection{Cell viability assays}

Cell viability on materials was tested using MTS assay CellTiter 96® AQueous One Solution Cell Proliferation assay (Promega) according to manufacturer's protocol. $50 \mu \mathrm{l}$ of MTS were added in each sample cultured with $250 \mu \mathrm{l}$ of medium, incubating for 3 hours and then recording the absorbance at 490 nm.

LIFE/DEAD Viability/Cytotoxicity assay Kit for Mammalian Cells (Invitrogen) discriminates live from dead cells by simultaneously staining with green-fluorescent calcein-AM (life cells) and red-fluorescent ethidium homodimer-1 (dead cells). Live/Dead assay was performed by adding $300 \mu \mathrm{l}$ of $4 \mu \mathrm{M}$ EthD-1 and $2 \mu \mathrm{M}$ of calcein AM in PBS per sample and incubated for 30-45 min at room temperature. Then, surfaces were observed with a Confocal TCS SP5 Upright from Leica Microsystems.

\subsection{Alkaline phosphatase assay}

Osteoblastic differentiation was evaluated through alkaline phosphatase (ALP) activity, with Abcam's Alkaline Phosphatase Assay Kit. This assay uses p-nitrophenyl phosphate (pNPP) as a phosphatase substrate which turns yellow when dephosphorylated (it turns to p-nitrophenol) by ALP. The resulting absorbance was measured at $405 \mathrm{~nm}$ using a scanning multi-well spectrophotometer.

\subsection{Cell morphology}

Phalloidin-Tetramethylrhodamine B isothiocyanate (Sigma-Aldrich) was used for determining the structure of the cytoskeleton by staining the F-actin. Cells were cleaned with PBS several times and fixed 10 min in $3.7 \%$ formaldehyde (Probus) solution in PBS. Then, cells were washed extensively again in PBS and permeabilized with $0.1 \%$ TRITON® X-100 (Sigma-Aldrich) in PBS 5 min. After that, cells were washed again in PBS and immersed in phalloidin (protected from the light) $40 \mathrm{~min}$ at room temperature. Cells were observed with a Leica Confocal TCS SP5 Upright.

\subsection{Statistical analysis}

Statistical analyses were performed by Mann-Whitney U test for group comparisons in the SPSS v.12.0 for Windows. All analyses were two-tailed, and p-values $<0.05$ were considered significant. 


\section{RESULTS}

\subsection{Coating characterization}

Figure 2 shows the FESEM micrographs showing the top surface morphology of CS: (i) CP-Ti coatings and (ii) NC-HA and (iii) C-HA coatings onto CP-Ti coatings. During the impact, the coarse feedstock Ti particles underwent plastic deformation and adhered to the Ti6Al4V alloy substrates. As it can be observed in table 1, the CS CP-Ti surfaces (Fig. 2a) show significant roughness due to the large particle size and relatively low particle flattening upon impact. As a consequence of the high roughness, a high waviness is noticeable. Additionally, it should be remarked that since the powder used has an irregular morphology, the titanium particles themselves show certain nanotexture on their surface, which might also play some role for cell attachment. These two contributions lead to high specific surface areas.

On the other hand, the fragmentation of the sintered HA feedstock powder results in a top surface morphology composed of submicron grains of polygonal shape (Fig. 2b), whereas the agglomerated powder leads to a more irregular and finer morphology (Fig. 2c). In both cases a decrease of surface roughness was observed, as HA layer smothered the groves and ridges of the CP-Ti surface. All roughness values parameters that were obtained by confocal microscopy were thus lower for the surfaces covered with HA compared to simply as-sprayed CS CP-Ti (Table 1). The nanotexturization given by the AFM measurements resulted in values of $\mathrm{Ra}=58.2 \mathrm{~nm}$ and $\mathrm{Ra}=39.1 \mathrm{~nm}$ for C-HA and NC-HA respectively, due to the larger micron-sized grains of the CS C-HA particles than the fine agglomerates of the CS NC-HA. This tendency had also been observed for the microroughness values. However, no AFM measurements were able to be performed on CS CP-Ti coatings.

Figure 3a shows the cross section of a CS CP-Ti coating. The use of coarse titanium particles leads to high surface roughnesses up to $\mathrm{Ra}=40 \pm 13 \mu \mathrm{m}$ (Table 1). Good particle cohesion at the contact points could be observed (Fig. 3a). Figures $3 \mathrm{~b}$ and $3 \mathrm{c}$ show CS HA coatings onto CS CP-Ti coatings. It is observed that HA deposition covers the as-sprayed rough titanium surface (especially the valleys), with the aim to induce surface bioactivity by the deposition of about $\sim 50 \mu \mathrm{m}$ of HA.

The X-Ray diffraction (XRD) of both CS HA coatings is shown in figure 4. The CS NC-HA coating exhibits less intense and broader peaks due to its nanocrystalline microstructure. Previous analysis indicated that the CS NC-HA layer presents an amount of $86.58 \%$ of HA phase, $2.07 \%$ of monetite phase and $11.34 \%$ of amorphous phase, with a crystal size around 11nm [34]. Contrary, the XRD of C-HA surface shows narrow and intense peaks due to higher crystallinity; no amorphous phase was detected and showed a crystal size between $0.5-2 \mu \mathrm{m}$ [9]. Regarding surface wettability, CS CP-Ti coatings show hydrophilic behavior with a contact angle of $26 \pm 10^{\circ}$; in a previous work, this was compared with the angle of $33 \pm 2^{\circ}$ exhibited by a sandblasted Ti6Al4V alloy substrate [39]. After the deposition of the respective HA layers, the CS HA coatings show superhydrophilic behavior with a contact value angle of $0^{\circ}$.

\subsection{Osteoblast viability}


Results of Live/Dead assay are shown in figure 5. Overall, all coatings showed good viability and there is no evident number of dead cells at all times tested. Along culture periods, all coatings showed an increase of cell number along the surface. CS CP-Ti coatings showed significantly higher cell attachment on the surface at 1 day of cell culture (Fig. 5) leading to a faster start cell proliferation in comparison with CS NCHA coatings (Fig. 6). Hence, after 7 days of cell culture, significant cell proliferation was detected in CS CP-Ti coatings compared to HA coatings, being the CS NC-HA, the coating with the slowest proliferation rates (Fig. 6). At 14 days of cell culture, a rapid increase of cell proliferation was observed on HA coatings, especially on CS NC-HA coatings, achieving similar MTS values with CS CP-Ti coatings (Fig. 6).

\subsection{Osteoblast differentiation}

Osteoblastic function was evaluated by assessing the ALP activity (Fig. 7). HA coatings showed significantly lower ALP values in comparison with CS CP-Ti coatings at all times of culture. Comparing HA coatings, the C-HA coatings showed higher values compared to NC-HA coating although differences were attenuated at 14 days of cell culture.

\subsection{Osteoblast morphology}

Osteoblast cells were stained with Phalloidin in order to analyze the cell morphology at 1, 7 and 14 days of cell culture (Fig. 8). At 1 day of culture, the osteoblast cells onto CS CP-Ti coatings showed spread and flattened morphology with extended filopodia on all directions, s showing good attachment onto the surface. On the other hand, cells onto HA coatings tended to have round like-shape morphology with a few extended filopodia and elongated cytoplasm in one direction. CS NC-HA coatings presented the highest amount of round like-shape morphology cells in comparison with CS C-HA coatings. At 7 days of cell culture, cells onto CS CP-Ti and CS NC-HA coatings showed the same cell spread and flattened morphology with elongated filopodia connecting each other, as well as proper cell attachment onto the surfaces. Otherwise, cells on CS C-HA coatings still spreading their cytoplasm and attaching onto the surface. At 14 days of cell culture, CS CP-Ti and CS NC-HA coatings showed full surface covered by osteoblast with extended and flattened actin filopodia. Cells onto CS C-HA coatings showed osteoblastic morphology and good attachment; although the number of cells was less in comparison with CS CP-Ti and CS NC-HA coatings due to the slow cell attachment at culture initiation.

\section{DISCUSSION}

Many works within the literature can be found concerning the influence of the composition, crystallinity, surface roughness and wettability (surface energy) on the in vitro performance. Most of the studies tend to make comparisons based on one of such effects and trying to keep the others fixed. However, their precise control is usually difficult and the synergic effect of all these variables must be taken into consideration when discussing the in vitro responses of osteoblastic cells. Here, the three cold sprayed coatings that cells are in contact with (one titanium and two hydroxyapatites), differ in composition, roughness, crystallinity and wettability, and the discussion will be addressed trying to evaluate the characteristic that plays a more predominant role.

\subsection{Coating deposition}


During the impact with the substrate, ductile particles in cold spray undergo plastic deformation and adhere to the surface. The kinetic energy of the particles, supplied by the expansion of the gas, is converted into plastic deformation energy allowing bonding between particles [40]. Here, the titanium coarse particles adhere one to the other, being able to build up porous coatings [39]. Particles impact at very high strain rates and, due to the very short impact time, the process is considered to be adiabatic and the deformation is highly localized [41].

The deposition of the sintered HA powders (C-HA) was observed to proceed through pore collapse, fragmentation and densification as well as grain refinement [9,34]. Particles crushed and deposited onto the substrate via first, by collapse and fracturing the necks resulting from the sintering process and further, by dynamic fragmentation into small subgrains [9]. Contrary, the agglomerated structure of the NC-HA powder deposited through the compaction of the nanocrystalline grains within the particle upon impact [34]. A finer surface structure was obtained from NC-HA coatings in comparison with the C-HA ones.

\subsection{Morphological cell aspects according to cell viability and proliferation}

In general, microstructured surfaces can affect cell morphology and cytoskeletal structure, while nanostructured surfaces can influence cell functions, including proliferation, differentiation and alignment $[20,42]$. In this study, we observed spread cell morphology with polygonal flattened cytoplasm and extended filopodia on CS CP-Ti coatings whereas round like-shape morphology was observed in cells on HA coatings, particularly at 1 day of cell culture. Hence, cells on CS CP-Ti coatings showed faster adhesion in comparison with cells on CS HA coatings. However, at 7 days of cells culture, cells on all tested coatings showed a flattened cytoplasm with extended filopodia.

The rough CP-Ti surface produced by CS were fully composed by a diversity of random groves and ridges, showing a quite complex surface composed by micro-roughness topography produced by the use of coarse CP-Ti particles plus certain nanofeatures provided by the irregular morphology of the CP-Ti feedstock particles. A more uniform submicrometer morphology was that one produced by the impact of hydroxyapatite particles when impacting onto the titanium coating, resulting in the rupture of such HA particles as explained in the previous section. By looking at the cell morphology onto such surfaces, they appear to anchor better to the coatings without HA, possibly with a big influence on topographic results [43, 44]. CS C-HA coatings presented lower roughness parameters than CS CP-Ti coatings, and CS NCHA coatings even lower, which seems to be in agreement to the general trend of MTS and ALP assays, indicating a role of the topography. CS NC-HA particles were more effective on smoothing the CS CP-Ti surfaces by covering the random groves and ridges through a tamping effect of the particles [34], while the C-HA particles just covered the surface [9]. The Live/Dead assay did not show significant cell mortality for any of the three surfaces tested in the present work along all days of cell culture, but it was observed that cells proliferated faster onto the CS CP-Ti coatings than CS HA surely by their faster attachment efficiency on the material at the culture initiation.

Different surface topography results in different surface wettability and this wettability, in turn, depends also on the composition of the material. The results showed a hydrophilic behavior for CS CP-Ti coatings but a superhydrophilic behavior for CS HA coatings. In all cases, the liquid drop penetrated into the surface invaginations (Wenzel regime). Wenzel relationship includes the influence of the surface roughness on 
enhancing the wettability caused by the chemistry of the surface [45]. According to Wenzel law, the increased roughness of the microstructured surfaces (and thus enhanced effective surface areas) makes the hydrophobic surface even more hydrophobic and hydrophilic surface even more hydrophilic [46]. A drop placed on a rough surface will spread until it finds its equilibrium configuration characterized by a $\theta^{*}$ (possibly different from the measured angle $\theta$ ). Therefore, the Wenzel regime was applied according to equation 1:

$$
\begin{gathered}
\text { (1) } \cos \theta^{*}=r * \cos \theta \\
\text { (2) } r=1+\operatorname{Sdr} / 100 \\
\text { (3) } \operatorname{Sdr}=\frac{\text { (Textured surface area) }-(\text { Cross section area) }}{\text { (Cross section area) }}
\end{gathered}
$$

In which " $\theta *$ " is defined as the Wenzel angle, " $\theta$ " as the measured (also known Young) angle and " $r$ " is the roughness ratio (eq.2), defined by $\mathrm{Sdr}$, the area factor (eq.3) [47]; thus, the contact angle obtained by Wenzel correction was of $\theta: 24 \pm 16^{\circ}$. The high protrusions and microporosity of CS CP-Ti coatings clearly enhanced surface area, thus surface wettability. After the deposition of the HA particles, the surface area increased even more due to the porous nature of these layers, leading to a superhydrophilic surface behavior $[48,49,50]$. Specially, nanophase materials have higher numbers of atoms at the surface compared with bulk, greater areas of increased surface defects, and larger proportions of delocalized surface electrons. Such altered surface properties will influence initial protein interactions that control subsequent cell adhesion [51]. Xiong et al. [50] studied in vitro the crystallinity, surface micro-roughness and surface energy of nano-hydroxyapatite coatings obtained by hydrothermal process at different temperatures. The increase of temperature between from 80 to $160^{\circ} \mathrm{C}$ led to an increase of surface energy and HA crystallinity but a decrease in surface roughness, thus an increase of cell proliferation. However, fewer amounts of cells were found at $200{ }^{\circ} \mathrm{C}$, where the highest crystallinity and less surface roughness was found, but also lower surface energy. Results suggested surface energy (surface wettability) as a characteristic parameter influencing the in vitro proliferation of osteoblast-like cells was predominant over the crystallinity and surface micro-roughness of the nano-hydroxyapatite coatings. Our results showed, at a later stage of culture, a faster cell proliferation of cells onto superyhydrophilic HA coatings, especially on the NC-HA coatings that had the finest structure. Osteoblast functions onto grains smaller than $100 \mathrm{~nm}$ are significantly enhanced compared with larger grains leading to a faster bone ingrowth [26], and also provide high surface area which induces better bioactivity than coarse crystals [25]. The nanocrystalline structure of NC-HA coatings together with the small amount of ACP, allows physiologically HA precipitation and enhance the bone ingrowth [12].

Results suggested that cell attachment is highly influenced by surface topography features. However, once cells are well attached, cell proliferation is mainly influenced by surface wettability (or surface energy) followed by composition and surface topography.

\subsection{Cell differentiation}


Rough surfaces have been well-known recognized for its stimulation on osteoblast differentiation [52]. A combination of micro- and nano-roughness has been demonstrated to enhance cell differentiation in CS CP-Ti coatings. The introduction of nanoscale structures in combination with micro-/submicro-scale roughness improves osteoblast differentiation and local factor production, which, in turn, indicates the potential for improved implant osseointegration in vivo [53]. Moreover, it is reported that nano-to micro surface roughness $(\mathrm{Ra}=10.57 \mu \mathrm{m})$ enhance cell mineralization [52]. Actually, the high-waviness profile of those coatings increases the free surface contact area between host-bone and the implant [43].

The production of porous coatings with an open-cell structure has been reported as an advantage, with a pore size up to 150 and a $60-65 \%$ of macroporosity [54]; this results in enhanced bone ingrowth and a good mechanical fixation. Rough CS CP-Ti coatings produced by CS surface showed the highest cell differentiation values in comparison with CS HA coatings. The lowest ALP activity in HA could be influenced by the time that cells take to attach to the substrate and to get the osteoblastic-like shape by an optimal cell function. Moreover, the lower number of viable cells on HA coatings compared to Ti during all culture time would explain the low ALP levels observed in HA cultures.

\section{CONCLUSIONS}

Poor cell attachment of cells onto HA coatings would lead to a delay in the start of proliferation until acquire an osteoblastic-like morphology, observed at 7 days of cell culture. Hence, cell response strongly depends on cell attachment, which is suggested to be mainly favored by surface topography. From 7 days of cell culture, osteoblast cells on CS HA surfaces achieved faster cell proliferation, especially for the CS NC-HA coatings. The finer porous nanocrytalline structures of CS NC-HA coatings, with smaller pore size and larger pore number, lead to an increase of surface area, as well as surface wettability, thus enhancing cell proliferation. On the other hand, ALP activity was found to be higher for CS CP-Ti coatings, likely due to the higher number of viable cells observed during all the culture time. Further long-time in-vitro test should be performed due to the time that cells required to start proliferating.

It is important however to emphasize that the results here presented do not indicate in general terms that porous titanium coatings are better than hydroxyapatite ones; it is just indicating that this happens for the specific coating conditions here used. It cannot be dealt in general terms since each time that not only the spraying technique, but also the spraying conditions or the feedstock powder are modified, the surface conditions (roughness, wettability...) can change considerably.

\section{Acknowledgments}

This work was supported by the Red Temática de Investigación Cooperativa en Envejecimiento y Fragilidad (RETICEF; RD12/0043/0022) and FEDER funds.

The authors also want to thank the Spanish MINECO for financial support through project MAT201346755-R and the Generalitat de Catalunya for the project 2014 SGR 1558, and University of Barcelona for the award of a scholarship that has helped the development of this research.

\section{REFERENCES}


[1] L. Le Guéhennec, A. Soueidan, P. Layrolle, Y. Amouriq, Surface treatments of titanium dental implants for rapid osseointegration, Dent Mater. 23 (2007) 844-854.

[2] S. Amin Yavari, J. van der Stok, Y.C. Chai, R. Wauthle, Z. Tahmasebi Birgani, P. Habibovic, M. Mulier, J. Schrooten, H. Weinans, A.A. Zadpoor, Bone regeneration performance of surface-treated porous titanium, Biomater. 35 (2014) 6172-6181.

[3] J.D.Bobyn, R.M. Pillar, H.U. Cameron, G.C.Weatherly, The optimum pore size for the fixation of porous-surfaced metal implants by the ingrowth of bone, Clin Orthop Relat Res. 150 (1980) 263-70.

[4] E.M. Lotz, R. Olivares-Navarrete, S. Berner, B.D. Boyan, Z. Schwartz, Osteogenic Response of Human MSCs and Osteoblasts to Hydrophilic and Hydrophobic Nanostructured Titanium Implant Surfaces, J Biomed Mater Res. 140A (2016) 3137-3148.

[5] G. Orsini, B. Assenza, A. Scarano, M. Piattelli, A. Piattelli, Surface analysis of machined versus sandblasted and acid-etched titanium implants, Int J Oral Maxillofac Implants. 15 (2000) 779-784.

[6] A. Verket, H. Tiainen, H.J. Haugen, S.P. Lyngstadaas, O. Nilsen, J.E. Reseland, Enhanced Osteoblast Differentiation on Scaffolds Coated with $\mathrm{TiO} 2$ Compared to $\mathrm{SiO} 2$ and $\mathrm{CaP}$ Coatings, Biointerphases. 7 (2012) 1-10.

[7] S. Minagar, J. Wang, C.C. Berndt, E.P. Ivanova, C. Wen, Cell response of anodized nanotubes on titanium and titanium alloys: Cell Response of Anodized TITANIA Nanotubes, J Biomed Mater Res A. 101A (2013) 2726-2739.

[8] B.-D. Hahn, D.-S. Park, J.-J. Choi, J. Ryu, W.-H. Yoon, K.-H. Kim, C. Park, H.-E. Kim, Dense Nanostructured Hydroxyapatite Coating on Titanium by Aerosol Deposition, J Am Ceram Soc. 92 (2009) $683-687$.

[9] N. Cinca, A.M. Vilardell, S. Dosta, A. Concustell, I. Garcia Cano, J.M. Guilemany, S. Estradé, A. Ruiz, F. Peiró, A New Alternative for Obtaining Nanocrystalline Bioactive Coatings: Study of Hydroxyapatite Deposition Mechanisms by Cold Gas Spraying, J Am Ceram Soc. 99 (2016) 1420-1428.

[10] J.H. Lee, H.L. Jang, K.M. Lee, H.-R. Baek, K. Jin, K.S. Hong, J.H. Noh, H.-K. Lee, In vitro and in vivo evaluation of the bioactivity of hydroxyapatite-coated polyetheretherketone biocomposites created by cold spray technology, Acta Biomater. 9 (2013) 6177-6187.

[11] K. de Groot, J.G.C. Wolke, J.A. Jansen, Calcium phosphate coatings for medical implants, Proceedings of the Institution of Mechanical Engineers, Part H: Journal of Engineering in Medicine. 212 (1998) 137147.

[12] L. Sun, C.C. Berndt, K.A. Gross, A. Kucuk, Material fundamentals and clinical performance of plasma-sprayed hydroxyapatite coatings: A review, J Biomedl Mater Res. 58 (2001) 570-592.

[13] N.P. Hailer, S. Lazarinis, K.T. Mäkelä, A. Eskelinen, A.M. Fenstad, G. Hallan, L. Havelin, S. Overgaard, A.B. Pedersen, F. Mehnert, J. Kärrholm, Hydroxyapatite coating does not improve uncemented 
stem survival after total hip arthroplasty!: An analysis of 116,069 THAs in the Nordic Arthroplasty Register Association (NARA) database, Acta Orthop. 86 (2015) 18-25.

[14] J. Wang, J. DiPietro, M. Bostrom, B. Nestor, D. Padgett, G. Westrich, Clinical and Radiographic Outcomes with a Hydroxyapatite and Porous Coated Cup Design, Adv Orthop Surgery. 2014 (2014) 1-5.

[15] M. Stilling, O. Rahbek, K. Søballe, Inferior Survival of Hydroxyapatite versus Titanium-coated Cups at 15 Years, Clinical Orthopaedics and Related Research®. 467 (2009) 2872-2879.

[16] K.A. Gross, C.C. Berndt, H. Herman, Amorphous phase formation in plasma-sprayed hydroxyapatite coatings, J Biomed Mater Res. 39 (1998) 407-414.

[17] J. Fernández, M. Gaona, J.M. Guilemany, Effect of Heat Treatments on HVOF Hydroxyapatite Coatings, J Therm Spray Technol. 16 (2007) 220-228.

[18] M.Gaona (2007) Recubrimientos biocompatibles obtenidos por Proyección Térmica y estudio in vitro de la función osteoblástica. $\mathrm{PhD}$ thesis. Universitat de Barcelona.

[19] D.M. Dohan Ehrenfest, P.G. Coelho, B.-S. Kang, Y.-T. Sul, T. Albrektsson, Classification of osseointegrated implant surfaces: materials, chemistry and topography, Trends in Biotechnol. 28 (2010) 198-206.

[20] L. Le Guehennec, M.-A. Lopez-Heredia, B. Enkel, P. Weiss, Y. Amouriq, P. Layrolle, Osteoblastic cell behaviour on different titanium implant surfaces, Acta Biomater. 4 (2008) 535-543.

[21] R.A. Gittens, T. McLachlan, R. Olivares-Navarrete, Y. Cai, S. Berner, R. Tannenbaum, Z. Schwartz, K.H. Sandhage, B.D. Boyan, The effects of combined micron-/submicron-scale surface roughness and nanoscale features on cell proliferation and differentiation, Biomater. 32 (2011) 3395-3403.

[22] M. Wei, H.-M. Kim, T. Kokubo, J.H. Evans, Optimising the bioactivity of alkaline-treated titanium alloy, Mater Sci Eng C. 20 (2002) 125-134.

[23] M.Mucalo, Hydroxyapatite (HAp) for Biomedical Applications. Elsevier 2015.

[24] D.J. Fernandes, C.N. Elias, R.Z. Valiev, Properties and Performance of Ultrafine Grained Titanium for Biomedical Applications, Materials Research. 18 (2015) 1163-1175.

[25] I. Mobasherpour, M.S. Heshajin, A. Kazemzadeh, M. Zakeri, Synthesis of nanocrystalline hydroxyapatite by using precipitation method, J Alloys Compds. 430 (2007) 330-333.

[26] T. Webster, Enhanced functions of osteoblasts on nanophase ceramics, Biomater. 21 (2000) 18031810 .

[27] J.M. Guilemany, N. Cinca, S. Dosta, I. Garcia-Cano. Intelectual Property: Feasibility of cold gas spraying to produce high roughness high porous titanium coatings for metallic prosthesis, Ref Number 1870 - . Universitat de Barcelona. Legal Deposit: 17/10/2014, Spain, 2014 
[28] T. Schmidt, F. Gärtner, H. Assadi, H. Kreye, Development of a generalized parameter window for cold spray deposition, Acta Mater. 54 (2006) 729-742.

[29] S. Dosta, M. Couto, J.M. Guilemany, Cold spray deposition of a WC-25Co cermet onto Al7075-T6 and carbon steel substrates, Acta Mater. 61 (2013) 643-652.

[30] J.-O. Kliemann, H. Gutzmann, F. Gärtner, H. Hübner, C. Borchers, T. Klassen, Formation of ColdSprayed Ceramic Titanium Dioxide Layers on Metal Surfaces, J Therm Spray Technol. 20 (2011) 292298.

[31] A.M. Vilardell, N. Cinca, A. Concustell, S. Dosta, I.G. Cano, J.M. Guilemany, Cold spray as an emerging technology for biocompatible and antibacterial coatings: state of art, J Maters Sci. 50 (2015) $4441-4462$.

[32] E. Kergourlay, D. Grossin, N. Cinca, C. Josse, S. Dosta, G. Bertrand, I. Garcia, J.M. Guilemany, C. Rey, First Cold Spraying of Carbonated Biomimetic Nanocrystalline Apatite on Ti6Al4V: PhysicalChemical, Microstructural, and Preliminary Mechanical Characterizations: First Cold Spraying of Carbonated Biomimetic Nanocrystalline, Adv Eng Mater. 18 (2016) 496-500.

[33] A. M. Vilardell, N. Cinca, S. Dosta, I. G. Cano, and J. M. Guilemany,"Multifunctional Miro-Nano Structured Hydroxyapatite Coatings OnttianumTi6Al4V for Biomedical Applications by Cold Gas Spray," Acta. de Protocolarización num., 809 (2015).

[34] A.M. Vilardell, N. Cinca, I.G. Cano, A. Concustell, S. Dosta, J.M. Guilemany, S. Estradé, A. RuizCaridad, F. Peiró, Dense nanostructured calcium phosphate coating on titanium by cold spray, J Eur Ceram Soc. 37 (2017) 1747-1755.

[35] M. Gardon, H. Melero, N. Garcia-Giralt, S. Dosta, I.G. Cano, J.M. Guilemany, Enhancing the bioactivity of polymeric implants by means of cold gas spray coatings: Enhancing The Bioactivity Of Polymeric Implants, J Biomed Mater Res B: Appl Biomater. 102 (2014) 1537-1543.

[36] A.C.W. Noorakma, H. Zuhailawati, V. Aishvarya, B.K. Dhindaw, Hydroxyapatite-Coated Magnesium-Based Biodegradable Alloy: Cold Spray Deposition and Simulated Body Fluid Studies, J Maters Eng Perf. 22 (2013) 2997-3004.

[37] X.B. Zheng, H. Ji, J.Q. Huang, C.X. Ding, Plasma sprayed TI and HA coatings: a comparative study between APS and VPS. Acta Metallurgica Sinica. 18 (2005) 339-344

[38] M. Nàcher, J. Aubia, S. Serrano, M.L. Mariñoso, J. Hernández, J. Bosch, A. Díez, J.M. Puig, J. Lloveras, Effect of cyclosporine A on normal human osteoblasts in vitro, Bone Miner. 26 (1994) 231-243.

[39] AM. Vilardell. Thesis dissertation. Functional coatings by cold spray for joint prosthesis. University of Barcelona, 2016.

[40] R. Ghelichi, M. Guagliano, Coating by the cold spray process: a state o the art. Fratt Integr Strutt 8 (2009) 30-44 
[41] G. Bae, S. Kumar, S. Yoon, K. Kang, H. Na, H-J. Kim, C. Lee, Bonding features and associated mechanisms in kinetic sprayed titanium coatings. Acta Mater 57 (2009) 5654-5666.

[42] H. Jeon, C.G. Simon, G. Kim, A mini-review: Cell response to microscale, nanoscale, and hierarchical patterning of surface structure: A Mini-Review, J Biomed Mater Res B Appl Biomater. 102 (2014) 15801594.

[43] J.I. Rosales-Leal, M.A. Rodríguez-Valverde, G. Mazzaglia, P.J. Ramón-Torregrosa, L. DíazRodríguez, O. García-Martínez, M. Vallecillo-Capilla, C. Ruiz, M.A. Cabrerizo-Vílchez, Effect of roughness, wettability and morphology of engineered titanium surfaces on osteoblast-like cell adhesion, Colloids Surf A. 365 (2010) 222-229.

[44] D. Khang, J. Lu, C. Yao, K.M. Haberstroh, T.J. Webster, The role of nanometer and sub-micron surface features on vascular and bone cell adhesion on titanium, Biomater. 29 (2008) 970-983.

[45] R.N. Wenzel. Resistance of solid surfaces to wetting by water. Ind. Eng. Chem Res. 1936;28: 988994.

[46] M. Kulkarni, Y. Patil-Sen, I. Junkar, C.V. Kulkarni, M. Lorenzetti, A. Iglic, Wettability studies of topologically distinct titanium surfaces. Colloids Surf. B. 129,(2015) 47-53

[47]http://www.biolinscientific.com/zafepress.php?url=\%2Fpdf\%2FAttension\%2FTheory\%20Notes\%2F

AT_TN_7_roughness.pdf

[48] G. Rosenman, D Aronov, Wettability engineering and bioactivation of hydroxyapatite nanoceramics. ChemInform. 38 (2007)

[49] D.Aronov, R.Rosen, E.Z.Ron, G.Rosenman, Tunable hydroxyapatite wettability: Effect on adhesion of biological molecules. Process Biochem. 41(2006) 2367-2372

[50] J. Xiong, Y. Li, P.D. Hodgson, C.Wen, In vitro osteoblast-like cell proliferation on nanohydroxyapatite coatings with different morphologies on a titanium-niobium shape memory alloy. J. Biomed. Res. 95(2010):766-73.

[51] M. Nelson, G. Balasundaram, T.J.Webster, Increased osteoblast adhesion on nanoparticulate crystalline hydroxyapatite functionalized with KRSR. Int J Nanomedicine. 1(2006) 339-349.

[52] E. Mariscal-Muñoz, C.A.S. Costa, H.S. Tavares, J. Bianchi, J. Hebling, J.P.B. Machado, U.H. Lerner, P.P.C. Souza, Osteoblast differentiation is enhanced by a nano-to-micro hybrid titanium surface created by Yb:YAG laser irradiation, Clin Oral Inv. 20 (2016) 503-511.

[53] R.A. Gittens, T. McLachlan, R. Olivares-Navarrete, Y. Cai, S. Berner, R. Tannenbaum, Z. Schwartz, K.H. Sandhage, B.D. Boyan, The effects of combined micron-/submicron-scale surface roughness and nanoscale features on cell proliferation and differentiation, Biomater. 32 (2011) 3395-3403.

[54] D. Qiu, M. Zhang, L. Grøndahl, A novel composite porous coating approach for bioactive titaniumbased orthopedic implants, J Biomed Mater Res A. 101A (2013) 862-872. 